\title{
Bioengineering and Biomedical Science
}

Yuehao Luo*

${ }^{1}$ Postdoctoral Research Scientist, School of Engineering and Applied Science, George Washington University, USA

*Corresponding author: Yuehao Luo, Postdoctoral Research Scientist, School of Engineering and Applied Science, George Washington University. USA, Tel: 001-5714396724; Email : luoyuehao@gwu.edu

Rec date: Jun 20, 2014, Acc date: Jun 22, 2014, Pub date: Jun 28, 2014

Copyright: @ 2014 Luo Y, et al. This is an open-access article distributed under the terms of the Creative Commons Attribution License, which permits unrestricted use, distribution, and reproduction in any medium, provided the original author and source are credited.

\section{Editorial}

\section{Fabrication of sharkskin surface in a large area by micro- rolling method}

Sharkskin has been fabricated by the direct bio-replicated imprinting method in previous studies, and the good drag-reducing effect has been validated in water tunnel. However, for the fluid surface larger than the shark's whole area, the splicing and affixing steps are absolutely necessary. One hand, the complexity of process will be increased significantly. The other hand, the phenomena of stress concentration produced by external flow will be produced on the jointing seams, especially for those which are perpendicular to the flowing direction, the jointing surface will be destroyed at a high speed of flowing, and perhaps, the drag-reducing efficiency can be decreased. Therefore, how to manufacture continuous vivid sharkskin in a large area has become an urgent problem to be resolved. For eliminating the influence of wedge angle on scale's back, the sputtering and photo lithography processes are put into application and the continuous vivid sharkskin with good forming effect is fabricated by rolling process at last.

\section{Numerical simulation of flow field on real sharkskin surface and exploring the drag reduction mechanism}

It is well known that sharkskin surface can effectively inhibit the occurrence of turbulence and reduce the wall fiction, and in order to understand the mechanism of drag reduction, the numerical simulation is a good attempt. In this project, the 3-dimensional highlyaccurate digital model is extremely built based on the biological template, and the turbulent flow on a real shark skin is simulated thoroughly and deeply, which explain the drag reduction mechanism of shark skin comprehensively.

\section{Experimental study of real shark skin for drag reduction effect in vacuole water tunnel}

For purpose of validating the accuracy and reliability of flow field numerical simulation on a real shark skin, the actual experiments are also performed subsequently. The real shark skin surface made of silicon rubber is fabricated by bio-replicated method firstly, and then the experiments in vacuole water tunnel, the drag reducing efficiency of real shark skin surface can be obtained by testing the force produced by the skins of real shark skin and smooth surface. And the results of numerical simulation and experiments are approximately consistent with each other, which verify the accuracy and reliability simultaneously.
The drag reduction efficiency of real shark skin surface can reach more than $12 \%$, and the attack angle of scales are the crucial factor to produce higher drag-reducing efficiency than simple straight grooves, and it can lead to the "back flowing" phenomena on the scale's surface.

\section{Application of bio-inspired drag-reducing technology in nature gas pipelining}

In order to increase the transmission capacity of gas pipelines, the internal coating technology has been vastly put into application, and a remarkable benefit has been achieved so far. However, with the reduction of wall roughness, the small convex parts are all completely submerged in the viscous sublayer, the gas pipeline becomes "hydraulic smooth pipe", even by smoothing the coating surface further, it is still very difficult to reduce wall friction. Therefore, in order to increase the transmission capacity on the basis of internal coating, the news methods and technologies should be researched and investigated, and perhaps, the bio-inspired drag reduction technology is a good attempt.

In the project, according to the actual transmitting circumstances of nature gas, the size and shape of bio-inspired drag-reducing surface is designed and analyzed firstly, and then the numerical simulation of flow field in nature gas pipeline is carried out deeply, and based on the characteristics and properties of the epoxy resin materials used for coating, the micro-rolling process is adopted, and the bio-inspired drag reduction pipelines are manufactured, and the field experiments with air and nature is performed finally.

The results of numerical simulation and experiments in air and nature gas are approximately consistent with each other, which verifies the feasibility of application bio-inspired drag reducing technology on nature gas transportation, and the drag reducing efficiency can reach more than $8 \%$.

\section{Manufacturing of different bio-inspired drag-reducing surfaces fitting to different circumstances}

According to the relevant literatures and researches, the uppermost factor affecting the drag-reducing efficiency is the non-dimension groove width $s+$ and the non-dimension groove height $h+$, which has the direct relationship with velocity, kinematic viscosity and so on. Therefore, the different bio-inspired surfaces fitting to different circumstances are manufactured by Ultrasonic Elliptical Vibration Cutting method, bio-replicated method, and 3-D dimension printing technology and so on, which expands the system of advanced manufacturing. 